\title{
A Multifunction Robot Based on the Slider-Crank Mechanism: Dynamics and Optimal Configuration for Energy Harvesting
}

\author{
A. Notué Kadjie a,1*, E. B. Tchawou Tchuisseu b,2 \\ a School of Wood, Water and Natural Resources, Faculty of Agronomy and Agricultural Sciences, Ebolowa Campus, \\ University of Dschang, P. O. Box 786, Ebolowa, Cameroon \\ ${ }^{\mathrm{b}}$ Laboratory of Rotational Laser Vibrometry, Institute of Thermomechanics of the Czech Academy of Sciences, \\ Dolejškova 1402, 18200 Praha 8-Libeň, Prague, Czech Republic \\ 1notuekajie@yahoo.fr; 2tchawou@gmail.com \\ * Corresponding Author
}

\section{ARTICLE INFO}

\section{Article history}

Received 18 July 2021

Revised 09 August 2021

Accepted 13 August 2021

Keywords

Slider-Crank Mechanism;

Chaos;

Robot;

Energy Harvesting

\section{ABSTRACT}

An electromechanical robot based on the modified slider-crank mechanism with a damped spring hung at its plate terminal is investigated. The robot is first used for actuation operation and for energy harvesting purposes thereafter. Mathematical modeling in both cases is proposed. As an actuator, the robot is powered with a DC motor, and the effect of the voltage supply on the whole system dynamics is found out. From the numerical simulation based on the fourth-order Runge-Kutta algorithm, results show various dynamics of the subsystems, including periodicity, multi-periodicity, and chaos as depicted by the bifurcation diagrams. Applications can be found in industrial processes like sieving, shaking, cutting, pushing, crushing, or grinding. Regarding the case of the robot functioning as an energy harvester, two different configurations of the electrical circuit for both single and double loops are set up. The challenge is to determine the best configuration for the high performance of the harvester. It comes from theoretical predictions and experimental data that the efficiency of the robot depends on the range values of the electrical load resistance $R_{L}$. The double loop circuit is preferable for the low values of $\mathrm{R}_{\mathrm{L}}<50 \mathrm{Ohm}$ ) while the single loop is convenient for high values of $\mathrm{R}_{\mathrm{L}}$ $\geq 50 \mathrm{Ohm}$.

\section{Introduction}

Modern industrial machinery needs to be supplied with appropriate mechanisms in order to improve its performance, accuracy and take the benefit on time, space, and input energy. One of the simplest and most common mechanisms is known as the slider-crank system. It takes the advantages of (i): its simultaneous rotation and translational motion when it is driven by a motor and finds the typical applications in petrol, fretsaws, gasoline, and diesel engines [1]. (ii): It is high coupling in nonlinear equations that can generate particular behaviors [2-5]. Depending on the desired application, the design of the traditional slider-crank mechanism 
changes in order to modify the repetitive and alternate translational motion of the slider since its motion is limited to a finite range. Among the methods used, the pneumatic system or spring connected to the rod is practiced [6]. In this case, the pneumatic cylinder is compressed or extended as the crank rotates continuously whilst the slider stays at a short duration at each stop. The other method is the use of a flexible rod with or without clearance, giving the opportunity to the whole system to operate at high speed [7-9]. Adding elastic components at the slider side complicates the study. But as indicated above, the elastic components may be of different origins, such as pneumatic response and flexible structures fixed on the slider end. However, the dynamics of the slider-crank mechanism with viscoelastic media and driven by a DC motor have not yet been studied in the literature.

Rotating and/or translational motion-driven energy harvesters are widely investigated [3, 10-11]. These particular types of displacement are more attractive by reason of their omnipresent origin which is related to human motion, the functioning of conventional machines, vibration in buildings, bridges, cars, trains, aircraft, ships, manufacturing tools, etc. [1]. However, the late challenge is to maximize the energy conversion from mechanical sources to electrical load even at low operational speed. Consequently, many research works were carried out in order to improve the output signal or to broaden the frequency response by (1) taking advantage of nonlinear phenomena [12-13]; (2) adding many oscillators with staggered resonances [14]; (3) modifying the electrical or mechanical part with the addition of specific components [4]. The situation where the electrical part of an energy harvester has two subcircuits such that one must configure them for the high performance of the harvester was not studied enough in the literature.

In this work, a model of a complex robot that uses simultaneously rotating and translational motion is considered. View as an actuator, it is supplied by a DC motor, and the first contribution is to analyze the dynamics of the whole system in order to find some suitable applications. On the other hand, the robot is considered an energy harvester. Its electrical part can take two configurations: the case where the rotating generator and the translational generator are placed in series and the case where the generators are wired in parallel with the load resistance. The contribution of the research is, through numerical simulations and experimental trials, to determine the appropriate configuration in which the electrical power is maximal, assuming that electrical signals are continuously in phase. The second contribution is to analyze theoretically the dynamical behavior of the robot based on the slider-crank mechanism when the external torque changes. This manuscript is organized as follows: Section 2 presents the description and the mathematical formulation of the device, considering the robot as an actuator and then as an energy harvester. In section 3, the analysis of the dynamical behavior of the slider-crank mechanism under constant electrical current excitation is carried out. Section 4 deals with the theoretical analysis of the response of the device under a constant excitation. Then, an experimental study is done, symmetric to the analytical model, and comes to validate the theoretical predictions. Section 5 concludes the work.

\section{Design and mathematical modeling}

\subsection{Architecture}

Fig. 1(a) shows the architecture of the electromechanical robot. It consists of a rotating wheel subjected directly to the constant excitation from a DC motor. The rotation motion is transmitted to a translational subsystem through a slider-crank mechanism offering synchronized rotating and translational motion.

In Fig. 1(b), the represented device is taken as an energy harvester, and the electrical part is formed by the coils with iron core placed between permanent magnets within the wheel and hung to the translational components. The relative mechanical displacements cause the magnetic flux variations within the corresponding coils which consequently, induce a voltage at 
its terminals. The energy harvester system is able to output one or two voltages depending on the way coils are associated in series or one loop circuit (see Fig. 1(c)) or in parallel (see Fig. $1(d)$ ). In both cases, the parameter values of the whole system (and their units) are listed in Table 1 and Table 2 .

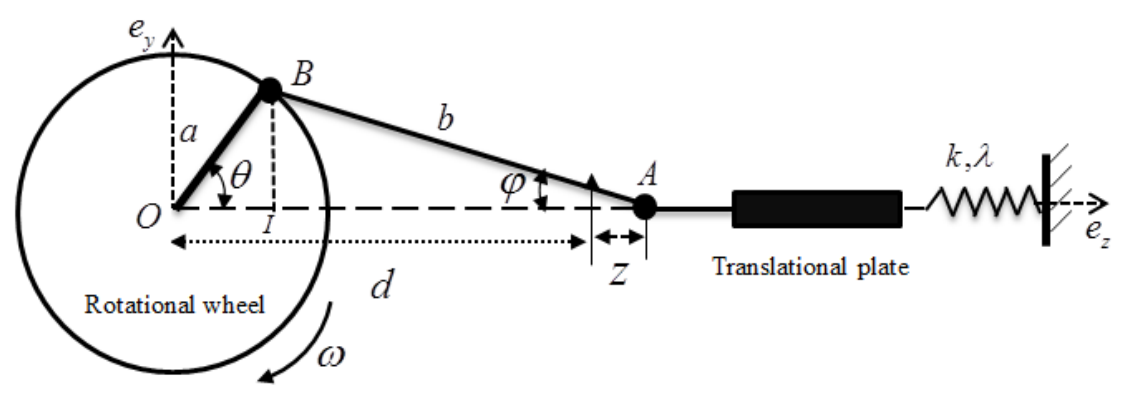

(a)

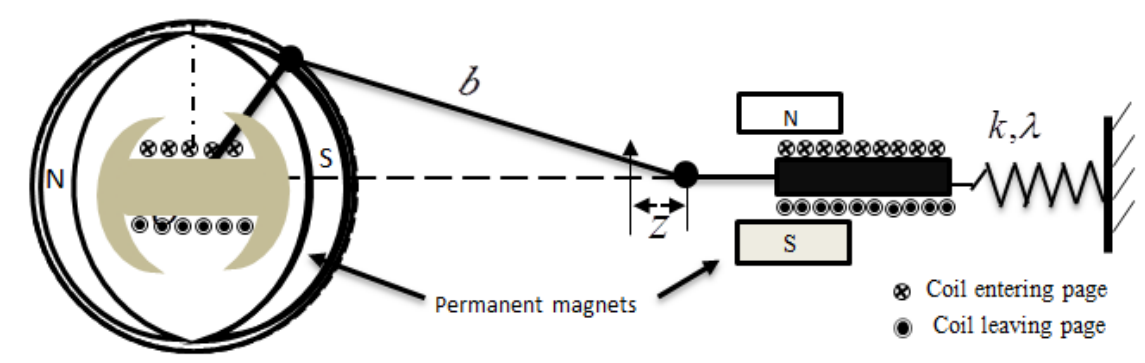

(b)



(c)

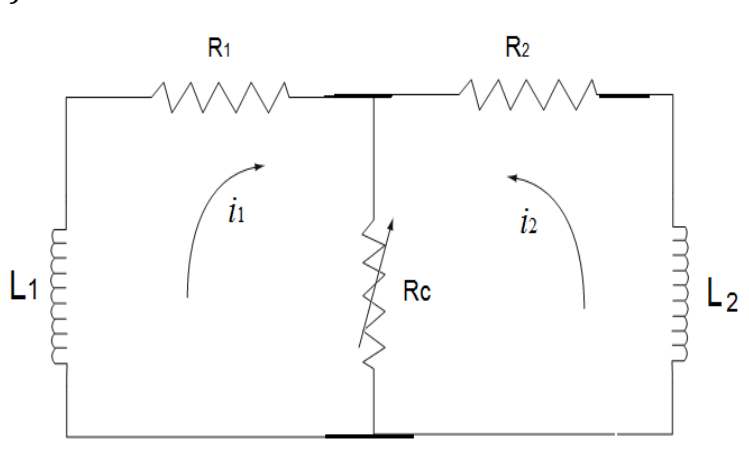

(d)

Fig. 1. Schematic representation of the electromechanical as actuator (a) as energy harvester (b) with the electrical part connected in series (c) or in parallel (d) with the load resistance Rc.

Table 1. Parameters of the translational subsystem

\begin{tabular}{ccc}
\hline Parameters & Value & Unit \\
\hline Crank radius: $\mathrm{a}$ & $3 \times 10^{-2}$ & $\mathrm{~m}$ \\
\hline Crank mass: $\mathrm{m}_{1}$ & 0.914 & $\mathrm{~kg}$ \\
\hline Rod mass: $\mathrm{m}_{2}$ & 0.3 & $\mathrm{~kg}$ \\
\hline Length of the crank: $\mathrm{b}$ & $21.7 \times 10^{-2}$ & $\mathrm{~m}$ \\
\hline Viscous damping: $\mathrm{C}_{1}$ & $1.5 \times 10^{-2}$ & $\mathrm{~N} . \mathrm{s} / \mathrm{m}$ \\
\hline Inductance: $\mathrm{L}_{1}$ & 150 & $\mathrm{mH}$ \\
\hline Electrical resistance: $\mathrm{R}_{\mathrm{C}}$ & Variable & $\Omega$ \\
\hline
\end{tabular}


Table 2. Parameters of the translational subsystem

\begin{tabular}{ccc}
\hline Parameters & Value & Unit \\
\hline Viscous damping: $\lambda$ & $1.5 \times 10^{-2}$ & $\mathrm{~N} . \mathrm{s} / \mathrm{m}$ \\
\hline Stiffness coefficient: $\mathrm{k}$ & 87.11 & $\mathrm{~N} / \mathrm{m}$ \\
\hline Inductance: $\mathrm{L}_{2}$ & 124 & $\mathrm{mH}$ \\
\hline Slider mass: $\mathrm{m}_{3}$ & 0.05 & $\mathrm{~kg}$ \\
\hline Electrical resistance: $\mathrm{R}_{2}$ & Variable & $\Omega$ \\
\hline
\end{tabular}

\subsection{Modeling of the multifunction robot as actuator robot driven by a DC motor}

\subsubsection{Modeling of the electrical part}

The equation that models the electrical part (motor) can be taken from classical textbooks or on research papers dealing with rotating electromechanical systems such as Ref. [6]. It can be written as:

$$
L_{m} \frac{d i}{d t}+R_{m} i+k_{e m} \frac{d \theta}{d t}=u_{0}
$$

where $i$ and $\theta$ are respectively the electric current and the angle formed by the crank direction and the $\left(O, e_{z}\right)$ axis. Variable $u_{0}$ is the constant input electrical voltage. The variables $L_{m}, R_{m}$, and $k_{e m}$ are respectively the inductance, the resistance, and the back electromotive constant of the DC motor.

\subsubsection{Modeling of the mechanical part}

The mechanical part of the system consists of the rotating and translational subsystem connected through a slider-crank mechanism (Fig. 1(a)). The length of the connecting rod length is assumed to be greater than the crank's length.

The mechanical part, viewed as connected to three mechanical subsystems:

- the crank strongly connected to the wheel moves with rotation motion,

- the rod which ensures the rigid link between the crank and the slider,

- the slider, which moves according to the translational motion.

It can be modeled as:

$$
J(\theta) \frac{d^{2} \theta}{d t^{2}}+\psi(\theta)\left(\frac{d \theta}{d t}\right)^{2}+C_{m} \frac{d \theta}{d t}+\kappa \cos \theta=T_{0}-k_{t} i-F_{m}(\theta)\left(1+\frac{a}{b} \cos \theta\right) a \sin \theta
$$

where $T_{0}$ is the torque from external mechanical excitation considered as constant in this work, $J(\theta)$ is the total inertia moment with respect to the angle $\theta$. Eq. (2) can be written as

$$
\begin{aligned}
J(\theta)= & \frac{1}{2} r_{g}^{2} m_{1}+\left[\left(a^{2}+\frac{a^{3}}{b} \cos \theta+\frac{a^{4}}{4 b^{2}} \cos ^{2} \theta\right) \sin ^{2} \theta+\frac{a^{2}}{4} \cos ^{2} \theta\right] m_{2} \\
& +\left[\left(a^{2}+\frac{2 a^{3}}{b} \cos \theta+\frac{a^{4}}{b^{2}} \cos ^{2} \theta\right) \sin ^{2} \theta\right] m_{3}
\end{aligned}
$$

Where $\psi(\theta)$ is the damping coefficient of the wheel that strongly depends on the angle $\theta$. Its expression is: 


$$
\begin{aligned}
\psi(\theta) & =\frac{3}{2}\left[-\left(\frac{a^{3}}{b}+\frac{a^{4}}{2 b} \cos \theta\right) \sin ^{3} \theta+\left(\frac{3}{4}+\frac{a^{2}}{4 b^{2}} \cos ^{2} \theta+\frac{a}{b} \cos \theta\right) a^{2} \sin 2 \theta\right] m_{2} \\
& +\frac{3}{2}\left[-2\left(\frac{a^{3}}{b}+\frac{a^{4}}{b} \cos \theta\right) \sin ^{3} \theta+\left(1+\frac{a^{2}}{b^{2}} \cos ^{2} \theta+\frac{2 a}{b} \cos \theta\right) a^{2} \sin 2 \theta\right] m_{3}
\end{aligned}
$$

Moreover, the coefficient

$$
\kappa=\frac{1}{2} m_{2} a g
$$

is relied on the potential energy of the system.

In (2), the terms $k_{t} i$ and $F_{m}(\theta)$ are respectively the electromotive force and the restoring force from the damped spring.

$$
F_{m}(\theta)=k\left(a \cos \theta+b-l_{0}\right)-\lambda\left(1+\frac{a}{b} \cos \theta\right) a \sin \theta
$$

The relation between the rotation angle $\theta$ and the slider displacement $\mathrm{z}_{3}$ is

$$
z_{3}=a \cos \theta+b \cos \varphi \approx a \cos \theta+b
$$

The details are presented in Appendix A. The reader can also report in [5] for other details.

Assuming that the dry friction forces and the magnetic losses are neglected, the resulting electromechanical equations which govern the system are:

$$
\left\{\begin{array}{l}
L_{m} \frac{d i}{d t}+R_{m} i+k_{e m} \frac{d \theta}{d t}=0 \\
J(\theta) \frac{d^{2} \theta}{d t^{2}}+\psi(\theta)\left(\frac{d \theta}{d t}\right)^{2}+C_{m} \frac{d \theta}{d t}+\kappa \cos \theta=T_{0}-\left(k_{t 1}+k_{t 2}\right) i-F_{m}(\theta)\left(1+\frac{a}{b} \cos \theta\right) a \sin \theta
\end{array}\right.
$$

\subsection{Modeling of the multifunction robot as an energy harvester}

\subsubsection{Equation formulation of the electrical part}

- Case of the single loop circuit

In the case of one loop, the inductance of the translational coil is disposed of in series with the inductance situated in the wheel (see Fig. 1(c)). Although the separate magnetic flux variation along with the associated coils, a unique electrical current flows in the wire and is used to power a load resistance $R_{L}$. The one-loop electrical system is described by the following equation [14-17]

$$
\dot{L} \frac{d i}{d t}+R_{e q} i=-\left(\boldsymbol{k}_{e \mathbf{1}} \frac{d \theta}{d t}+\boldsymbol{k}_{e 2} \frac{d z}{d t}\right)=-\boldsymbol{e}
$$

where $L=L_{1}+L_{2}$ is the equivalent inductance, $R_{e q}=R_{1}+R_{2}+R_{L}$ is the equivalent electrical resistance of the circuit and $e=e_{1}+e_{2}$ is the induced voltage form in rotating and translational motion, respectively. The variable $R_{L}$ is a load resistance. In (9), the coefficients $\boldsymbol{k}_{\boldsymbol{e} \mathbf{1}}$ and $\boldsymbol{k}_{\boldsymbol{e} \mathbf{2}}$ 
are the mechanical-to-electrical conversion coefficient while $\frac{d \theta}{d t}$ and $\frac{d z}{d t}$ are crank and slider velocity, respectively.

- Case of a double loop circuit

The coils can also be connected in parallel. Two output signals are available: It is the case of the double loops energy harvester. The rotating movement of the wheel and the translational coil induce different magnetic flux variations that create two electrical currents that power one electrical device, as shown in Fig. 1(b). According to Kirchhoff's law, the equation of this part considering the double loop case is:

$$
\begin{aligned}
& L_{1} \frac{d i_{1}}{d t}+\left(R_{1}+R_{L}\right) i_{1}=-\boldsymbol{k}_{\boldsymbol{e} \mathbf{1}} \frac{d \theta}{d t}=-\boldsymbol{e}_{\mathbf{1}} \\
& L_{2} \frac{d i_{2}}{d t}+\left(R_{2}+R_{L}\right) i_{2}=-\boldsymbol{k}_{\boldsymbol{e} 2} \frac{d z}{d t}=-\boldsymbol{e}_{2}
\end{aligned}
$$

The equation of the mechanical part is the one treated in section 2.2.2, the resulting electromechanical equations which govern the system are:

- for the single loop electrical circuit

$$
\left\{\begin{array}{l}
L \frac{d i}{d t}+R_{e q} i+k_{e} \frac{d \theta}{d t}=0 \\
J(\theta) \frac{d^{2} \theta}{d t^{2}}+\psi(\theta)\left(\frac{d \theta}{d t}\right)^{2}+C_{m} \frac{d \theta}{d t}+\kappa \cos \theta=T_{0}-\left(k_{t 1}+k_{t 2}\right) i-F_{m}(\theta)\left(1+\frac{a}{b} \cos \theta\right) a \sin \theta
\end{array}\right.
$$

- for the double loop electrical circuit

$$
\left\{\begin{array}{l}
L_{1} \frac{d i_{1}}{d t}+\left(R_{1}+R_{L}\right) i_{1}+k_{e 1} \frac{d \theta}{d t}=0 \\
L_{2} \frac{d i_{2}}{d t}+\left(R_{2}+R_{L}\right) i_{2}+k_{e 2} \frac{d z}{d t}=0 \\
J(\theta) \frac{d^{2} \theta}{d t^{2}}+\psi(\theta)\left(\frac{d \theta}{d t}\right)^{2}+C_{m} \frac{d \theta}{d t}+\kappa \cos \theta=T_{0}-k_{t 1} i_{1}-k_{t 2} i_{2}-F_{m}(\theta)\left(1+\frac{a}{b} \cos \theta\right) a \sin \theta
\end{array}\right.
$$

Where $k_{e 1}$ and $k_{e 2}$ are the electromotive force from the rotating wheel and translational plate, respectively.

One denotes

$$
\begin{gathered}
x=\frac{i}{i_{0}} ; x_{j}=\frac{i_{j}}{i_{0}} ; y=\frac{\theta}{\theta_{0}} ; \alpha_{m}=\frac{R}{L \omega_{0}} ; \beta_{m}=\frac{k_{e} \theta_{0}}{L i_{0}} ; T=\frac{T_{0}}{J(y) \omega_{0}^{2} \theta_{0}} ; \\
\tilde{\psi}(y)=\frac{\theta_{0} \psi(y)}{J(y)} ; \tilde{\kappa}=\frac{\kappa}{J(y) \omega_{0}^{2} \theta_{0}} ; \tilde{k}_{t i}=\frac{k_{t i}}{J(y) \omega_{0}^{2} \theta_{0}} ; \tilde{k}_{0}=\frac{k_{0}}{J(y) \omega_{0}^{2} \theta_{0}} ; \tilde{\lambda}_{0}=\frac{\lambda a}{J(y) \omega_{0}}
\end{gathered}
$$

The dimensionless electromechanical equations are written as: 
- for the circuit in series

$$
\left\{\begin{array}{l}
\dot{x}+\alpha_{m} x+\beta_{m 1} \dot{y}+\beta_{m 2} \dot{z}=0 \\
\ddot{y}+\tilde{\psi}(y) \dot{y}^{2}+\tilde{c} \dot{y}+\tilde{\kappa} \cos \left(\theta_{0} y\right)=T-\left(\tilde{k}_{t 1}+\tilde{k}_{t 2}\right) x-\tilde{\xi}(y)\left(1+\frac{a}{b} \cos \left(\theta_{0} y\right)\right) a \sin \left(\theta_{0} y\right)
\end{array}\right.
$$

- for the circuit in parallel

$$
\left\{\begin{array}{l}
\dot{x}_{1}+\alpha_{m 1} x_{1}+\beta_{m 1} \dot{y}=0 \\
\dot{x}_{2}+\alpha_{m 2} x_{2}+\beta_{m 2} \dot{z}=0 \\
\ddot{y}+\tilde{\psi}(y) \dot{y}^{2}+\tilde{c} \dot{y}+\tilde{\kappa} \cos \left(\theta_{0} y\right)=T-\left(\tilde{k}_{t 1}+\tilde{k}_{t 2}\right) x-\tilde{\xi}(y)\left(1+\frac{a}{b} \cos \left(\theta_{0} y\right)\right) a \sin \left(\theta_{0} y\right)
\end{array}\right.
$$

with

$$
\tilde{\xi}(y)=\tilde{k}_{0}\left(a \cos \left(\theta_{0} y\right)+b-l_{0}\right)-\tilde{\lambda}_{0}\left(1+\frac{a}{b} \cos \left(\theta_{0} y\right)\right) \dot{y} \sin \left(\theta_{0} y\right)
$$

Reference values are taken as $\theta_{0}=\pi \mathrm{rad}, i_{0}=1 \mathrm{~A}$ and $\omega_{0}=1 \mathrm{rad} / \mathrm{s}$

\section{Dynamics of the electromechanical robot under constant excitation}

This section deals with the dynamics of the whole system as the supplied voltage of the DC motor changes. The interest is to set the appropriate value in order to obtain suitable dynamics for the given application. Due to the complexity of the equations, this investigation is carried out numerically, and the main parameter is the DC motor excitation $\mathrm{u}_{0}$.

For this purpose, the fourth-order Runge-Kutta algorithm is used to solve the differential equations in (8). Fig. 2 presents the dynamics of the current, crank rotation velocity, angular displacement, and translational displacement $u_{0}=9.4 \mathrm{~V}$. One finds that a permanent rotation takes place. The current in the winding oscillates around its equilibrium value 0 (Fig. 2(a)), while the angular velocity oscillates around a non-zero value (Fig. 2(b)). These results are similar to those obtained by Jih-Lian Ha et al. [5] and Rong-Fong Fung et al. [6]. The angular displacement is increased with time and oscillating around an oblique line (Fig. 2(c)) while the translational displacement oscillates between its extremal values, which are $b+a=24.7 \mathrm{~cm}$ and $b-a=18.7 \mathrm{~cm}$ (Fig. 2(d)).

In order to delineate the domains for different types of dynamical behaviors, the bifurcation diagrams versus the amplitude $u_{0}$ are plotted for different values of $\mathrm{k}$ and $\mathrm{m}_{3}$. The bifurcation diagrams are obtained in the following manner:

- Eq. (8) is solved considering the mechanical excitation as a parameter.

- For each value of the parameter, the number of the period of the oscillation is numerically counted and represented in an $\left(u_{0}, d \theta / d t\right)$ axis.

- Each point in this $\left(u_{0}, d \theta / d t\right)$ axis represents an oscillation with a 1T-period. But, the superposition of points in the vertical direction means either a multi-periodicity or chaos. 

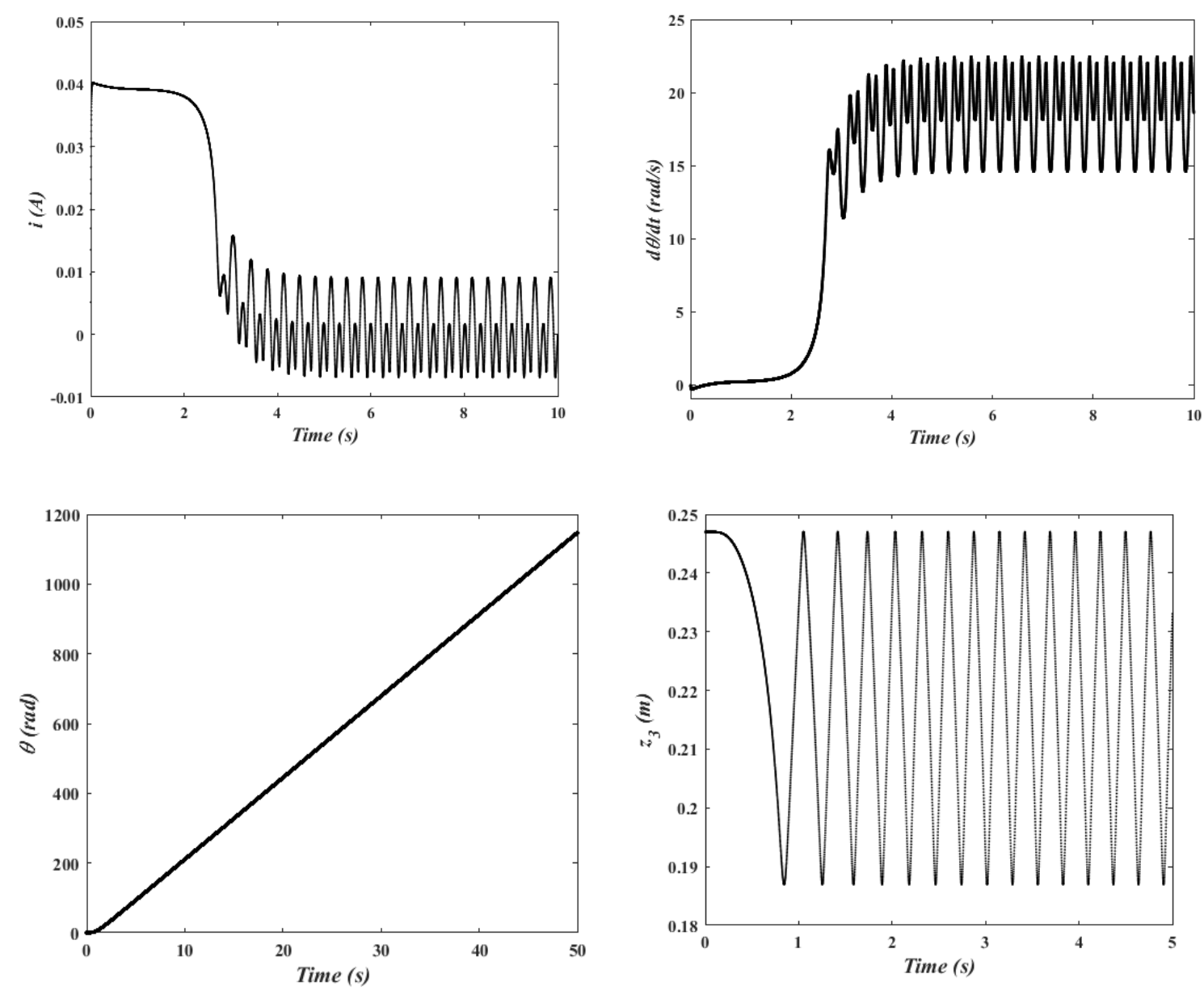

Fig. 2. Time traces of the electrical current (a), crank velocity (b), the total angle swept (c), and the slider position (d). These graphs are plotted with the parameters of Table 1 and 2 and $u_{0}=9.4 \mathrm{~V}, m_{3}=$

$$
0.5 \mathrm{~kg}, k=0.1 \mathrm{~N} / \mathrm{m} \text { and } \lambda=1.5 \times 10^{-2} \mathrm{~N} . \mathrm{m}^{-1} . \mathrm{s}
$$

For this purpose, Fig. 3 shows the bifurcation diagram taking the excitation amplitude as a parameter. It presents several dynamics of the crank velocity as $u_{0}$ change. More precisely, in Fig. 3(a), where $\mathrm{k}=0$ and $\mathrm{m}_{3}=0.5$, the oscillatory state with at least one period is observed for the small value of $u_{0}\left(u_{0}<20\right)$. Then, crank velocity behaves with chaos dynamics for the middle values of $u_{0}\left(20<u_{0}<27\right)$. Finally, the multi-periodicity follows. In other words, one assist at the multi-periodicity - chaos - multi periodicity scheme of the bifurcation diagram. This alternate scheme is also observed in Fig. $3(\mathrm{~b})$, plotted with $\mathrm{k}=0.25$ and $\mathrm{m}_{3}=0.5$. Nevertheless, it is distinguished by the narrower gaps between the alternating parts due to the influence of the dynamics imposed by the spring. In Fig. 3(c), where the spring is absent, a large gap exists between the multi periodicity - chaos - multi-periodicity patterns. But the increase of the value of the slider mass $\mathrm{m}_{3}$ tends to dissociate the chaotic area. The reverse situation is observed in Fig. 3(d), where the increase of the spring coefficient tends to enlarge the chaotic zone. 

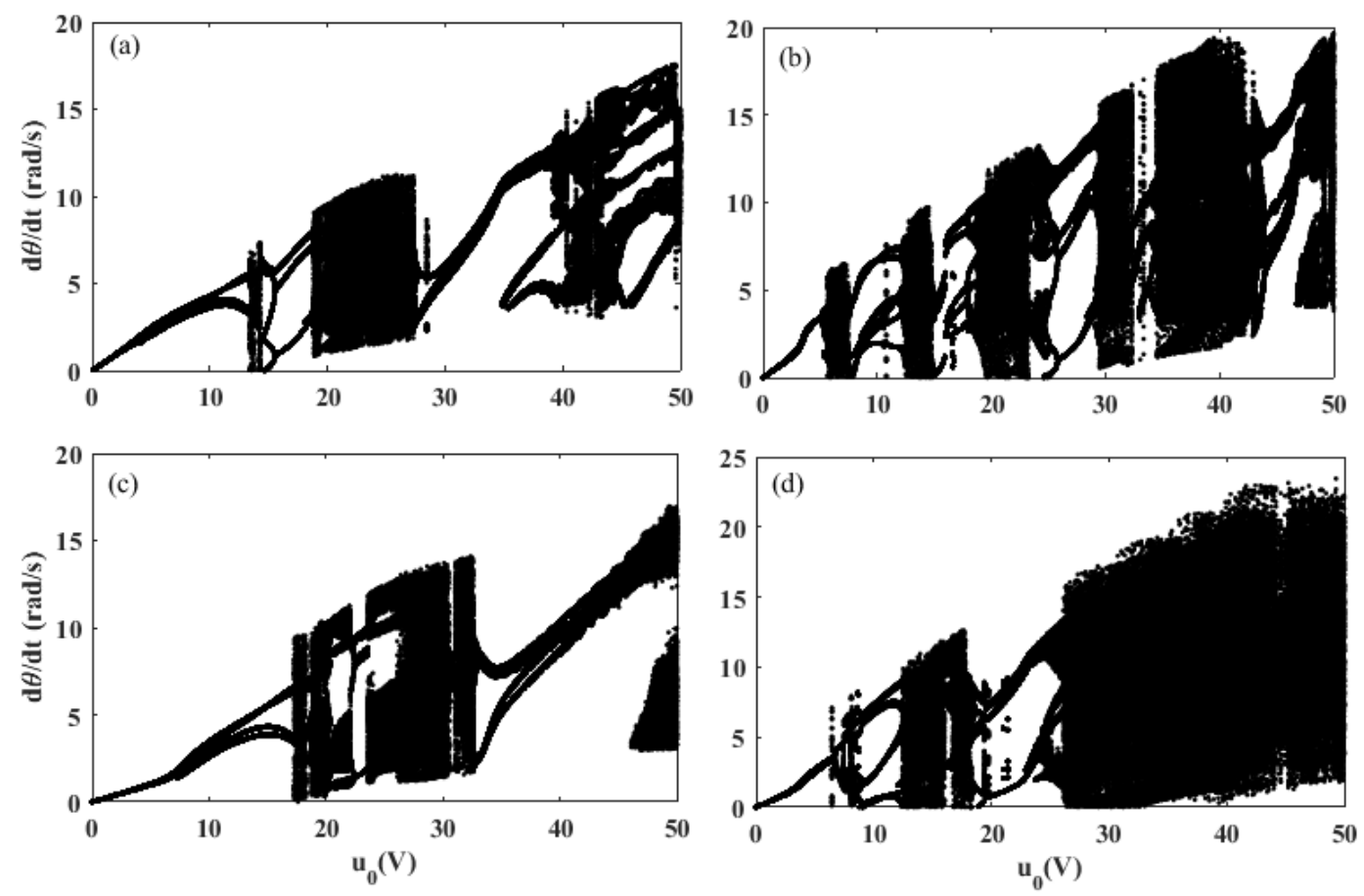

Fig. 3. Bifurcation diagram of the crank velocity as a function of the excitation amplitude $u_{0}$. These graphs are plotted using the parameters of Table 1 and Table 2. In (a), $k=0 \mathrm{~N} / \mathrm{m}$ and $m_{3}=0.5 \mathrm{~kg}$, In (b), $k=0.25 \mathrm{~N} / \mathrm{m}$ and $m_{3}=0.5 \mathrm{~kg}$, In (c), $k=0 \mathrm{~N} / \mathrm{m}$ and $m_{3}=1 \mathrm{~kg}$ and In (d), $k=0.25 \mathrm{~N} / \mathrm{m}$ and

$$
m_{3}=1 \mathrm{~kg} \text {. }
$$

\section{Electromechanical robot as an energy harvester}

\subsection{Electricity generation: a theoretical study}

This section focuses on the theoretical analysis and comparison of the generated electrical signal taking to the output of the energy harvester system in accordance with the external torque and load resistance having the electrical part of the system since (11) and (12) are not analytically solvable, this theoretical study is relied on the numerical simulation using the fourorder Runge Kutta algorithm considering the single case where the whole system is subjected to the constant and permanent torque. This situation depends on the disposition of the blades around the wheel, which are plunged within the laminar moving fluid. Alongside this work, the dimensionless variables are transformed into dimensional ones before being plotted in the Figures.

However, the rotating and the translational subsystems are linked with the rigid connection (a slider-crank mechanism). Consequently, their oscillatory response has the same frequency and constitutes a rotating-translational synchronized motion. This oscillatory state induces the sinusoidal output voltage available at the load resistance whatever the wiring of the electrical part, as shown in Fig. 4.

The time trace sketched on Fig. 4, considering some particular parameters, displays the shape of the expected electrical energy. This sinusoidal form can be treated with a specific electronic circuitry in order to convert it into the appropriate form easy to use or store. 


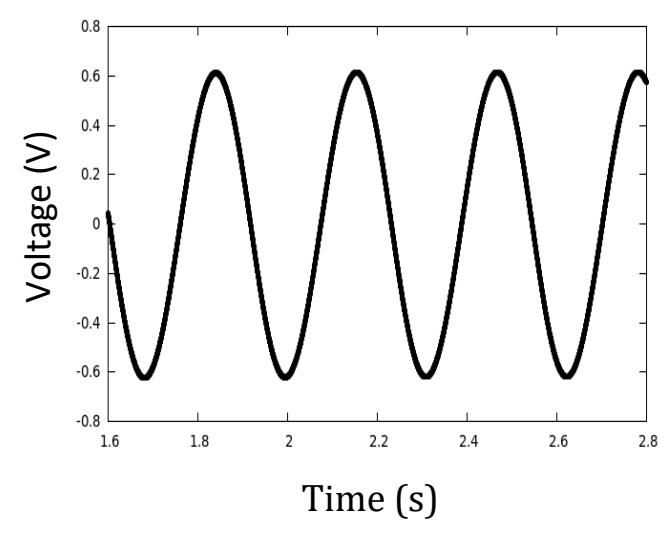

(a)

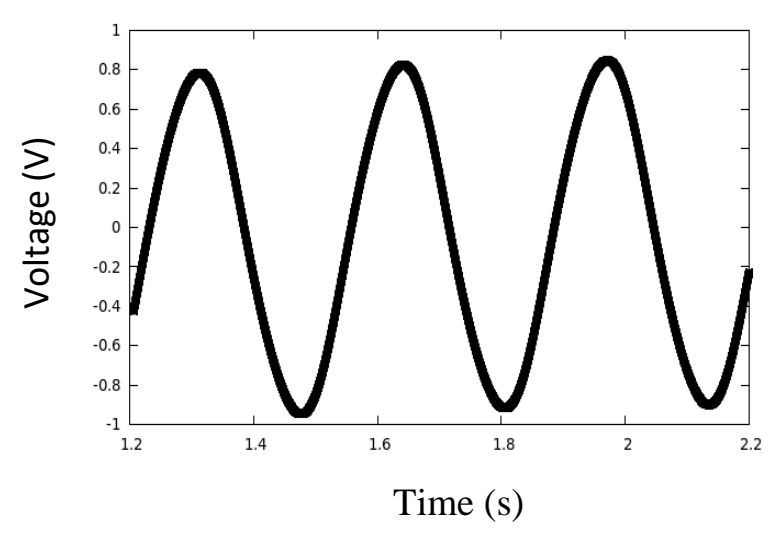

(b)

Fig. 4. Time trace of the available voltage to the load resistance. (a) One loop system, (b) double loop system. These graphs are plotted with the parameters of Tables 1 and 2 and $\mathrm{T}_{0}=10 \mathrm{Nm}, \mathrm{R}_{c}=47 \Omega$.

In Fig. 5, one plots the maximum of the voltage whilst the rotation rate changes. It is shown that the voltage increases substantially in the case of a single and double loop. This is due to the densification of the induction phenomena caused by the intense motion of the mechanical part and the linear behavior of the used inductances. Moreover, Fig. 5 also shows that whatever the considered frequency behavior of the mechanical part, the voltage provided by the double loop wire system is greater than that obtained with a single loop wire system. This observation can be justified by taking the association of the inductance and the internal resistance of the coils. The double loop output voltage is significant because of the low value of the inductance and the internal resistance. The low value of the coil's characteristics favors the electromagnetic conversion, firstly from the magnetic quantity to the electrical quantity, and secondly, the low internal transformation through Joule's effect.

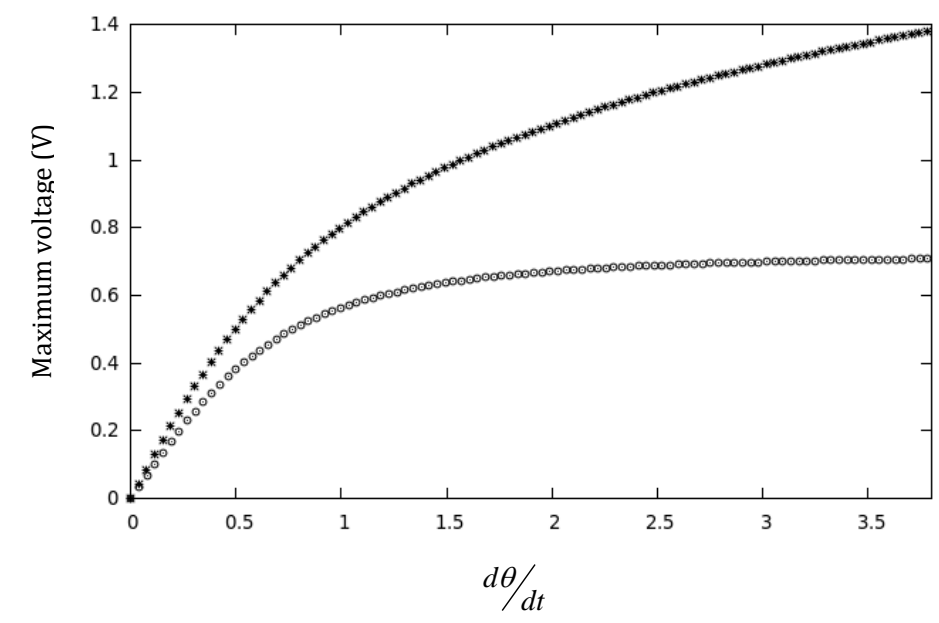

Fig. 5. Maximum voltage through the load resistance versus rotation rate. This graph is plotted with the parameters of Fig. $4\left(^{*}\right)$ double loop predictions $\left({ }^{\circ}\right)$ single-loop predictions.

The deep comparison is done rely on the electrical power delivered for each configuration case. Its expression is given by:

$$
\begin{gathered}
P_{1}=R_{C} i^{2} \\
P_{2}=R_{C}\left(i_{1}+i_{2}\right)^{2}
\end{gathered}
$$


Eq. (17) considers the electrical power in the case of a single loop, whereas (18) deals with the case of the double loop wire.

In Fig. 6, the maximum electrical power versus load resistance shows the peak resonance curve in each case. The peak value of the electrical power reaches when the resistance of the load approaches the resistance of the coil. Seeing that the equivalent value of the coils' resistance is different in accordance with the number of the mesh of the electrical part, the peak value of the power appears at the different resonance value.

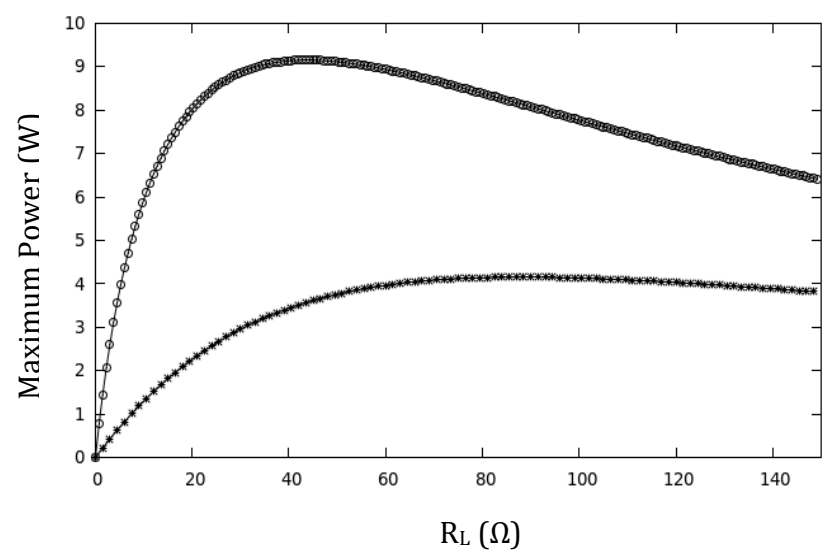

Fig. 6. Maximum of the electrical power vs. load resistance using the parameters of Fig. $4\left(^{*}\right)$ single loop harvesting $\left({ }^{\circ}\right)$ double loop harvesting.

\subsection{Results from an experimental prototype}

The device studied during the previous section is fabricated, and some experimental investigations were performed to compare with the theoretical predictions. These experimental trials concern the time histories of the response of the system under excitation, the maximum output voltage when the rotation rate sweeps a certain value and then, the electrical power delivered when the load resistance varies. With the intention to optimize the comparison between the theoretical simulation and the experimental results, the same approximate geometric parameter values are used and listed in Table 1 and Table 2.

Fig. 7 shows the mounted device and measurements apparatus. For the laboratory investigations, a DC motor is used to simulate the constant external torque excitation on which the fabricated device is tested. While the rotation of the wheel, a series of calibration experiments were performed to relate measured coil voltages using suitable apparatus (see Fig. 7).

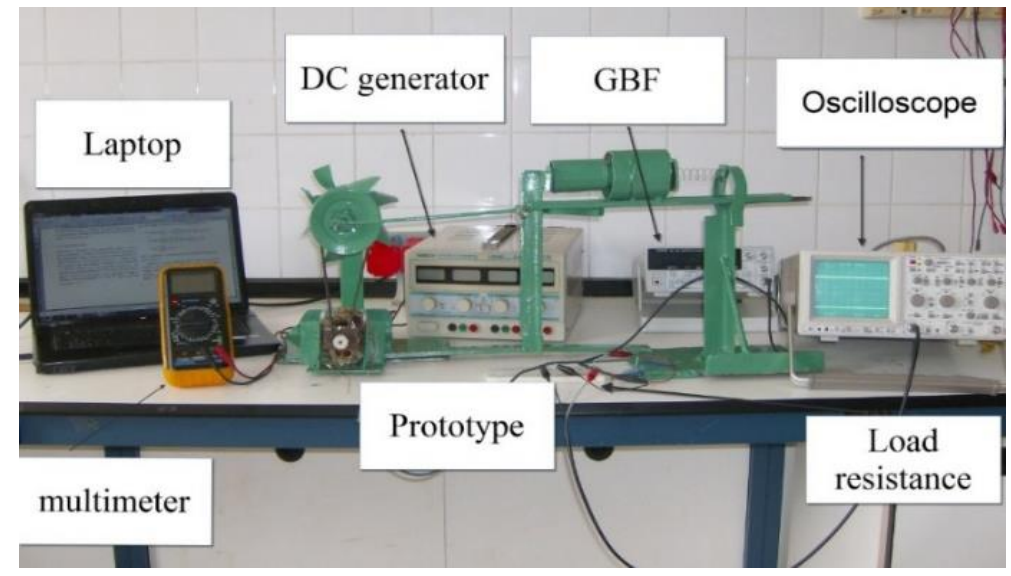

Fig. 7. Experimental setup 
The first data series from experiments is used to plot the time histories of the load resistance voltage. The plot is done separately in the single loop and double loop electrical system, as shown in Fig. 8(a) and Fig. 8(b).

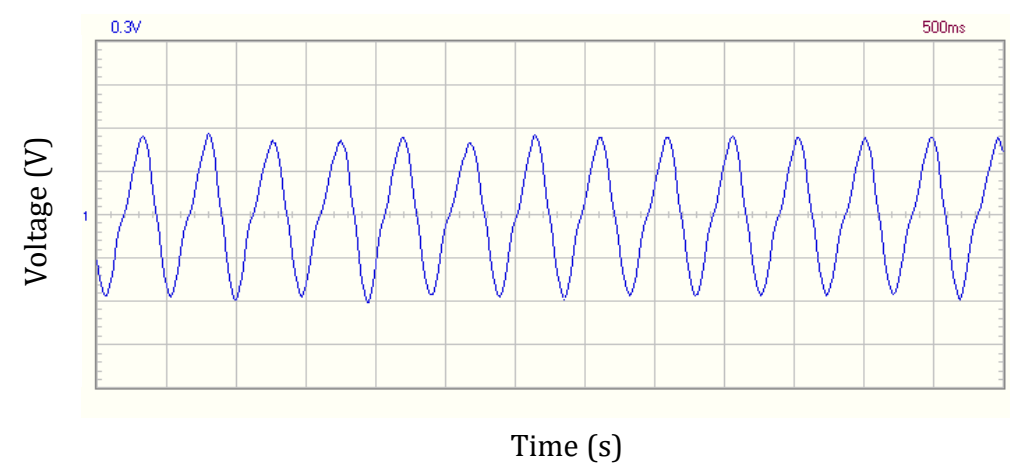

(a)

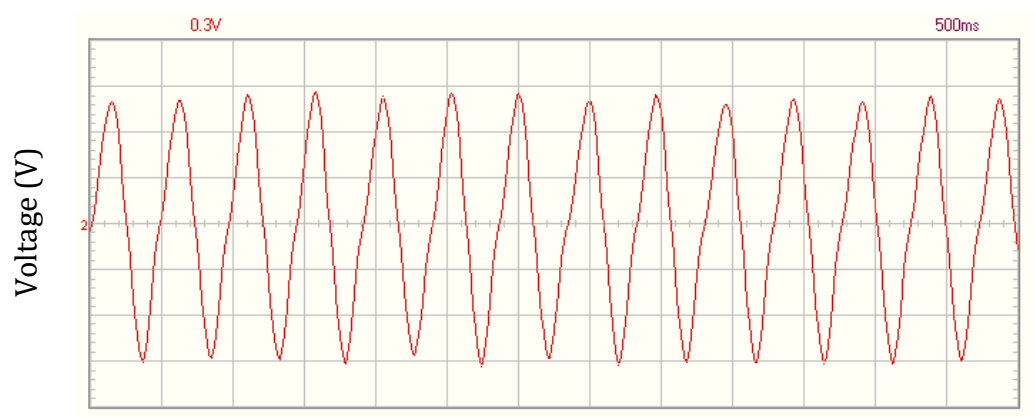

Time (s)

(b)

Fig. 8. Experimental time histories plot of the voltage at the resistance terminal (a) Single loop wire circuit (b) double loop wire circuit

As theoretical predictions, whatever the considered configuration, the output voltage oscillates during the time. However, Fig. 9 is plotted the theoretical and experimental value of the maximum of the output voltage as a function of the rotation rate in the double mesh case simultaneously.

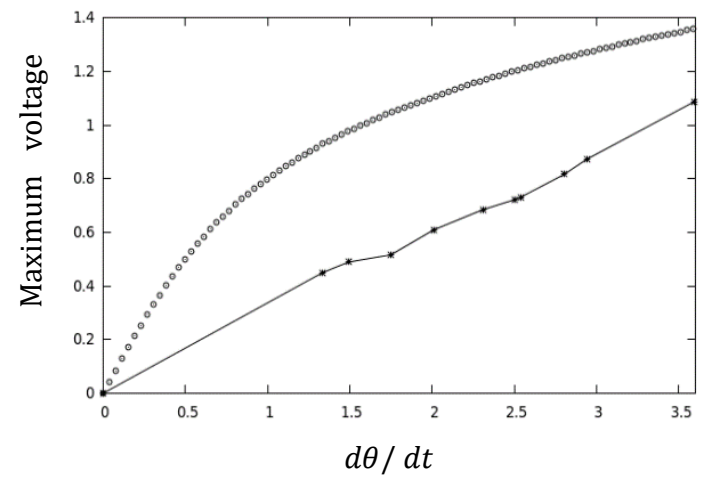

(a)

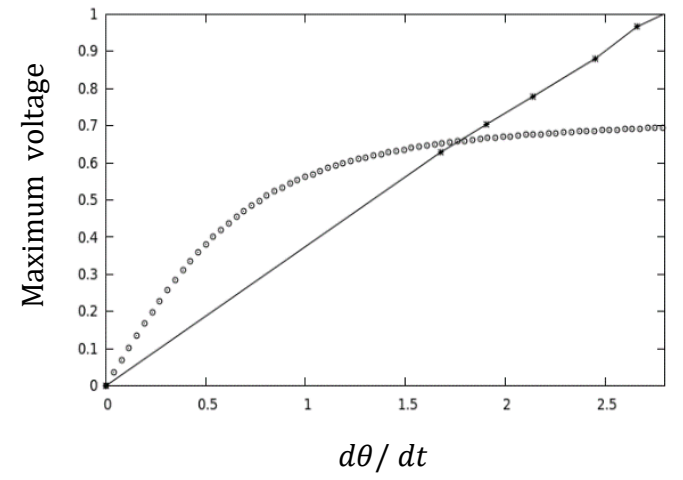

(b)

Fig. 9. Maximum output voltage as a function of the rotation rate in (a) the double loop configuration and (b) a single loop of the electrical part $\left({ }^{*}\right)$ experimental plot $\left({ }^{\circ}\right)$ theoretical predictions

It results that Fig. 9 presents the same qualitative behavior. This remark can be made in Fig. 9(b), where the single mesh case is considered. The observed gap between the theoretical and experimental plots can be due to the approximate used values. This gap also appears in Fig. 
10(a) and Fig. 10(b), where the comparison between the simulation method and experimental data are plotted. Finally, Fig. 11 shows that the electrical power is greater in the double loop case for the low value of the load resistance. But when the value of the load resistance is large, the single loop case is preferable to use.

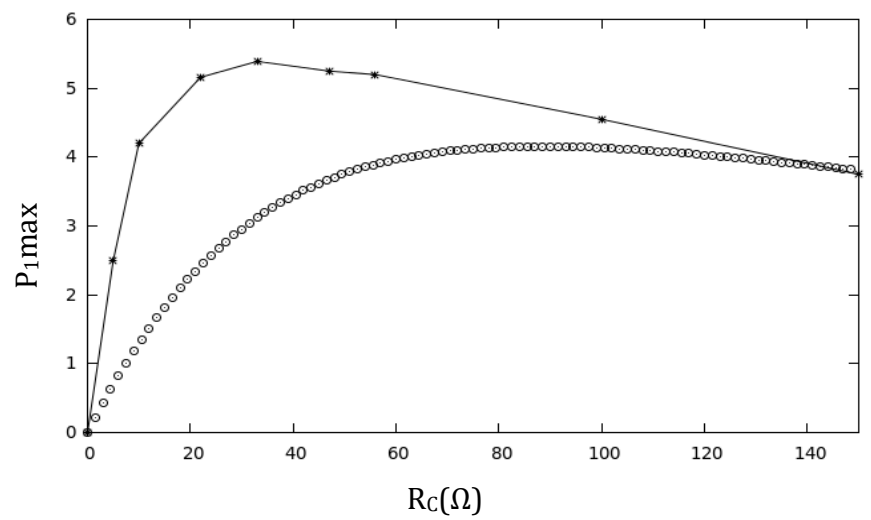

(a)

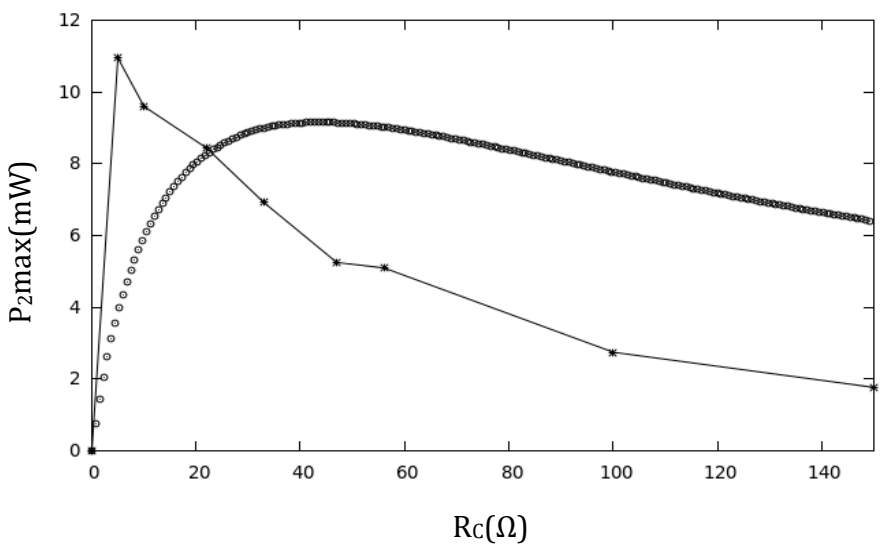

(b)

Fig. 10. Maximum output power vs. load resistance $\left({ }^{*}\right)$ experimental plot $\left({ }^{\circ}\right)$ theoretical predictions (a) Single loop (b) double loop

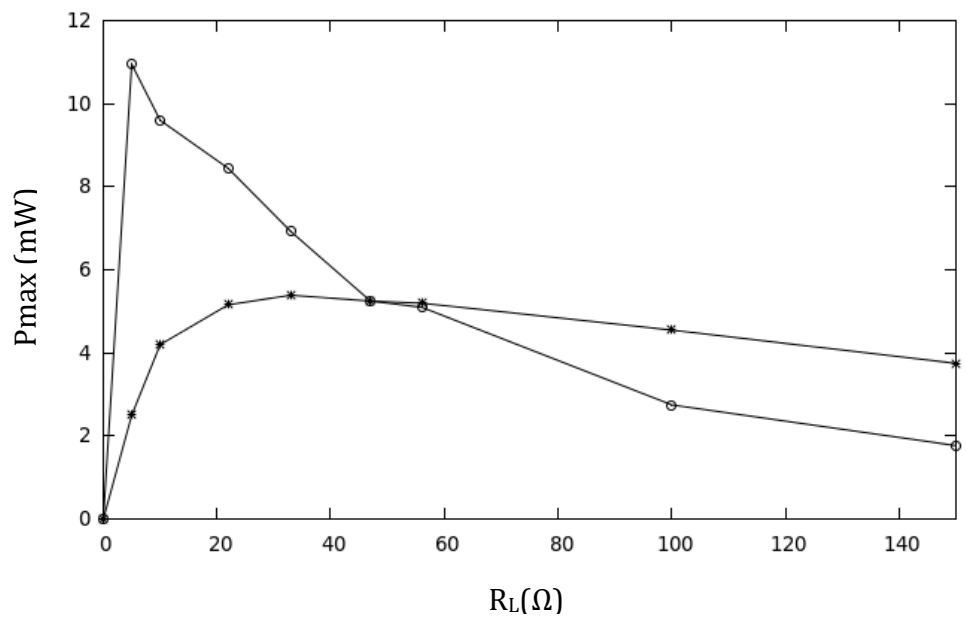

Fig. 11. Comparison of the experimental maximum output power vs. load resistance $\left({ }^{*}\right)$ one mesh wiring $\left({ }^{\circ}\right)$ two meshes wiring 


\section{Conclusion}

This work has dealt with the study of a robot based on the slider-crank mechanism displaying two functions, namely actuation, and energy harvesting operation. The modeling of the robot has been provided and equations traducing its dynamics established in both cases. Looking at the influence of the DC-driven source on the whole actuator robot dynamics, it has first come that the electrical current, the crank velocity, the total angle swept, and the slider position present a regular dynamical behavior as expected according to the literature review. A deep look at the crank velocity when the DC excitation varies has revealed complex dynamics leading to different regimes (periodicity, multi-periodicity, and chaos). It has also been seen in bifurcation diagrams that the domain corresponding to each regime can be enlarged or reduced by a particular choice on the spring coefficient and the slider mass value. Taking the robot as an energy harvester system, theoretical and experimental works have been carried out in order to determine the best configuration for the system to be more efficient. The studies have been devoted to the harvester robot with a single loop and a double loop electric circuit. It has come that the high performance of the system depends on the range values of the electrical load resistance. Also, it has been noted that the use of a double loop circuit should consider low values of the load resistance while the use of a single loop circuit is suitable to high values of $\mathrm{R}_{\mathrm{L}}$. One can consider in future work the case where the excitation is periodic, like a sinusoidal signal or square signal. In this case, an analytical approach can be envisaged for others comparison

\section{Acknowledgment}

The authors also thank Dr. Murielle Vanessa TCHAKUI for valuable discussions.

\section{Appendix}

According to Fig. 1(a), the center of mass of the crank is spotted in the $O, e_{y}, e_{z}$ axis by:

$$
y_{1}=0 \text { and } z_{1}=0
$$

The center of mass of the rod is spotted by:

$$
y_{2}=\frac{b}{2} \sin \varphi \text { and } z_{2}=a \cos \theta+\frac{b}{2} \cos \varphi
$$

The center of mass of the slider is spotted by:

$$
y_{3}=0 \text { and } z_{3}=a \cos \theta+b \cos \varphi
$$

In the triangle $\mathrm{OAB}$, the following relation is verified:

$$
a \sin \theta=b \sin \varphi
$$

The kinetic energy of the whole system is:

$$
T=T_{1}+T_{2}+T_{3}
$$

where $\left(T_{i}\right)_{i=1,2,3}$ are the kinetic energy of the crank, rod, and slider, respectively.

$$
T_{1}=\frac{1}{4} m_{1} r_{g}^{2} \dot{\theta}^{2}
$$




$$
\begin{aligned}
& T_{2}=\frac{1}{2} m_{2}\left(\dot{y}_{2}^{2}+\dot{z}_{2}^{2}\right)=m_{2} \dot{\theta}^{2}\left[\frac{1}{8} a^{2} \cos ^{2} \theta+\frac{1}{2}\left(a^{2} \sin ^{2} \theta+\frac{a^{3}}{b} \sin ^{2} \theta \cos \theta\right)\right] \\
& T_{3}=\frac{1}{2} m_{3}\left(\dot{y}_{3}^{2}+\dot{z}_{3}^{2}\right)=m_{3} \dot{\theta}^{2}\left[\frac{a^{4}}{2 b^{2}} \sin ^{2} \theta \cos ^{2} \theta+\frac{1}{2} a^{2} \sin ^{2} \theta+\frac{a^{3}}{b} \sin ^{2} \theta \cos \theta\right]
\end{aligned}
$$

The potential energy of the system is

$$
V=\frac{1}{2} m_{2} g a \sin \theta
$$

The Lagrangian of the system is, considering Eqs. (A5)-(A9), given as:

$$
L=T-V
$$

The generalized impulsion is expressed as:

$$
P=\frac{\partial L}{\partial \dot{\theta}}
$$

The Hamiltonian of the system is:

$$
H=\frac{1}{2} \frac{P^{2}}{\xi(\theta)}+\frac{1}{2} m_{2} g a \sin \theta
$$

The equation of the mechanical part is derived from Eq.(A13)

$$
\left\{\begin{array}{l}
\dot{P}=-\frac{\partial H}{\partial \theta}+F_{a} \\
\dot{\theta}=\frac{\partial H}{\partial P}
\end{array}\right.
$$

with $\sqrt{b^{2}-a^{2} \sin ^{2} \theta} \approx b$

\section{References}

[1] R. F. Fung and C. F. Chang, "Force/motion sliding mode control of three typical mechanisms," Asian Journal of control, vol. 11, pp. 196-210, March 2009. https://doi.org/10.1002/asjc.96

[2] R. F. Fung, Lin FJ, J. S. Huang and Y. C. Wang, "Application of the sliding mode control with a low pass filter to the constantly rotating slider-crank mechanism," Jpn Soc Mech Eng, Ser C, vol. 40, n ${ }^{\circ}$, pp. 717-722, 1997. https://doi.org/10.1299/jsmec.40.717

[3] D. Belato, H.I. Weber, J.M. Balthazar, D.T. Mook, "Chaotic vibrations of a nonideal electromechanical system," Int Journal of Solids and Structures, vol. 38, pp.1699-1706, 2001. https://doi.org/10.1016/S0020-7683(00)00130-X

[4] D. 0 Tcheutchoua Fossi and P. Woafo, "Dynamical behaviors of a plate activated by an induction motor," Journal of Sound and Vibration, vol. 329, pp. 3507-3519, 2010. https://doi.org/10.1016/j.jsv.2010.03.017 
[5] J. L. Ha, R. F. Fung, K. Y. Chen and S. C. Hsien, "Dynamic modeling and identification of a slidercrank mechanism," Journal of Sound and Vibration, vol. 289, pp. 1019-1044, 2006. https://doi.org/10.1016/j.jsv.2005.03.011

[6] R. F. Fung, C. L. Chiang and S. J. Chen, "Dynamic modelling of an intermittent slider-crank mechanism," Applied Mathematical Modelling, vol. 33, pp. 2411-2420, 2009. https://doi.org/10.1016/j.apm.2008.07.004

[7] I. Khemili and L. Romdhane, "Dymanic analysis of a flexible slider-crank mechanism with clearance," European Journal of Mchanics A/Solids, vol. 27, pp. 882-898, 2009. https://doi.org/10.1016/j.euromechsol.2007.12.004

[8] R. F. Fung, "Dynamic responses of the flexible connecting rod of a slider-crank mechanism with time-dependent boundary effect". Computers and Structures, Vol. 63, n 1, pp. 79-90, 1997. https://doi.org/10.1016/S0045-7949(96)00333-1

[9] S. R. Hsieh and S. W. Shaw, "The dynamic stability and nonlinear resonance of a flexible connecting rod: continuous parameter model," Nonliear Dynamics, vol. 4, pp. 573-603, 1993. http://dx.doi.org/10.1007/BF00162233

[10] S. Roundy, P. K. Wright and J. Rabaey, "A study of low level vibrations as a power source for wireless sensor nodes," Comput. Commun, vol. 26, pp. 1131-1144, 2003. https://doi.org/10.1016/S0140-3664(02)00248-7

[11] P.D. Mitcheson, T.C. Green, E.M. Yeatman and A.S. Holmes, "Architectures for vibration-driven micro power generators," J.Microelectromech. Syst., vol. 13, pp. 429-440, 2004. https://doi.org/10.1109/JMEMS.2004.830151

[12] T. V. Buren and G. Troster, "Design and optimization of a linear vibration-driven electromagnetic micro-power generator," Sensors and Actuators A, vol. 135, pp. 765-775, 2007. https://doi.org/10.1016/j.sna.2006.08.009

[13] S. Kulkarni, E. Koukharenko, R. Torah and J. Tudor, "Design, fabrication and test of integrated macro-scale vibration-based electromagnetic generator," Sensors and Actuators A Physical, vol.145, pp. 336-342, 2007. https://doi.org/10.1016/j.sna.2007.09.014

[14] B. P. Mann and B. A. Owens, "Investigations of a nonlinear energy harvester with a bistable potential well," Journal of Sound and Vibration, vol. 329, pp. 1215-1226, 2010. https://doi.org/10.1016/j.jsv.2009.11.034

[15] G.T. Oumbe Tekam, E. B. Tchawou Tchuisseu, C.A. Kitio Kwuimy and P. Woafo, "Analysis of an electromechanical energy harvester system with geometric and ferroresonant nonlinearities," Nonlinear Dynamics, vol. 76, pp. 1561-1568, January 2014, https://doi.org/10.1007/s11071-0131228-6

[16] A. Notue Kadjie, I. Kemajou and P. Woafo, "Control of an electromechanical pendulum subjected to impulse disturbances using the Melnikov theory approach," Journal of Mechanical Science and Technology, vol. 32, pp. 865-874, February 2018. https://doi.org/10.1007/s12206-018-0137-x

[17] R. T. Fotsa, A. R. Tchamda, A. S. K. Tsafack and S. T. Kingni, "Microcontroller Implementation, Chaos Control, Synchronization and Antisynchronization of Josephson Junction Model," International Journal of Robotics and Control Systems, vol. 1, no. 2, pp. 198-208, June 2021. https://doi.org/10.31763/ijrcs.v1i2.354 